\title{
BMJ Open Association of food industry ties with findings of studies examining the effect of dairy food intake on cardiovascular disease and mortality: systematic review and meta-analysis
}

\author{
Nicholas Chartres (D) , ${ }^{1}$ Alice Fabbri, ${ }^{2}$ Sally McDonald, ${ }^{1}$ Joanna Diong, ${ }^{3}$ \\ Joanne E McKenzie, ${ }^{4}$ Lisa Bero (iD ${ }^{1}$
}

To cite: Chartres N, Fabbri A, McDonald S, et al. Association of food industry ties with findings of studies examining the effect of dairy food intake on cardiovascular disease and mortality: systematic review and meta-analysis. BMJ Open 2020;10:e039036. doi:10.1136/ bmjopen-2020-039036

- Prepublication history and additional materials for this paper are available online. To view these files, please visit the journal online (http://dx.doi. org/10.1136/bmjopen-2020039036).

Received 01 April 2020 Revised 13 0ctober 2020 Accepted 25 0ctober 2020

Check for updates

(C) Author(s) (or their employer(s)) 2020. Re-use permitted under CC BY-NC. No commercial re-use. See rights and permissions. Published by BMJ.

For numbered affiliations see end of article.

Correspondence to

Dr Lisa Bero;

lisa.bero@sydney.edu.au

\section{ABSTRACT}

Objective To determine if the association of dairy foods with cardiovascular disease (CVD) outcomes differs between studies with food industry ties versus those without industry ties. To determine whether studies with or without industry ties differ in their risk of bias.

Eligibility criteria We included cohort and case-control studies that estimated the association of dairy foods with CVD outcomes in healthy adults.

Information sources We searched eight databases on 1 February 2019 from 2000 to 2019 and hand searched reference lists.

Risk of bias We used the Risk of Bias in Non-Randomised Studies-of Exposure tool.

Included studies 43 studies ( 3 case-controls, 40 cohorts).

Synthesis of results There was no clear evidence of an association between studies with industry ties $(1 / 14)$ versus no industry ties (8/29) and the reporting of favourable results, risk ratio $(\mathrm{RR})=0.26(95 \% \mathrm{Cl} 0.04$ to $1.87 ; n=43$ studies) and studies with industry ties (4/14) versus no industry ties (11/29) and favourable conclusions, $\mathrm{RR}=0.75$ (95\% $\mathrm{Cl} 0.29$ to $1.95 ; \mathrm{n}=43$ ).

Studies with industry sponsorship, $(\mathrm{HR}=0.78 ; \mathrm{n}=3$ studies) showed a decreased magnitude of risk of CVD outcomes compared with studies with no industry sponsorship ( $\mathrm{HR}=0.97 ; \mathrm{n}=18$ ) (ratio of HRs $0.80(95 \% \mathrm{Cl}$ 0.66 to 0.97$) ; p=0.03$ ).

Strengths and limitations of evidence Every study had an overall high risk of bias rating; this was primarily due to confounding.

Interpretation There was no clear evidence of an association between studies with food industry ties and the reporting of favourable results and conclusions compared with studies without industry ties. The statistically significant difference in the magnitude of effects identified in industry-sponsored studies compared with non-industry-sponsored studies, however, is important in quantifying industry influence on studies included in dietary guidelines.

PROSPERO registration number CRD42019129659.
Strengths and limitations of this study

- This is the first systematic review and meta-analysis to evaluate the association of food industry ties (industry sponsorship and/or author conflicts of interest (COI)) with the results, conclusions and risk of bias of primary nutrition studies examining the association of dairy foods with cardiovascular disease outcomes and mortality.

- We conducted a comprehensive search and followed explicit and well-defined inclusion and exclusion criteria for the included studies.

- For studies missing a funding or author COI disclosure, we did not contact the authors; thus we may be underestimating the number of studies with industry ties.

- The tool that we used to assess the risk of bias is still under modification, however it is unlikely any future changes to the tool will affect the risk of bias ratings.

- We did not analyse studies of low-fat and full-fat dairy separately. Industry ties may have different effects on studies of low-fat or full-fat dairy foods.

\section{INTRODUCTION}

The effect of dairy foods on cardiovascular disease (CVD) is unclear. Recent systematic reviews and meta-analyses of observational studies have reported conflicting results between the association of total dairy consumption and risk of CVD, with some showing decreased risk and some showing no clear evidence. ${ }^{1-4}$ The beneficial effects of decreasing blood pressure, however, appear more consistent. ${ }^{4}$ Further, dairy intake recommendations made in dietary guidelines around the world vary. Although the Australian Dietary Guidelines concluded that there is a probable association between dairy food consumption and a reduced risk of cardiovascular events, ${ }^{6}$ recent 
amendments to the Eatwell guidelines by Public Health England recommend a significant reduction in the daily intake of dairy foods. ${ }^{7}$

Food industry sponsors and authors with a conflict of interest (COI) with the food industry may gain financially from finding that dairy foods have health benefits, since such a finding can be used to market dairy products. Such a driver may lead industry sponsors to magnify (or bias) the health benefits of dairy foods by influencing the research agenda, design and conduct of the study, or reporting of the results. ${ }^{8-11}$ Prior examinations of pharmaceutical and tobacco research have identified that even when controlling for methodological biases, studies sponsored by industry were more likely to have results that favoured the sponsor than studies with other sources of sponsorship. ${ }^{12-14}$

The effects of food industry sponsorship or author COI with the food industry on study results need further examination. ${ }^{15} \mathrm{~A}$ systematic review assessing the association of wholegrain foods with CVD and mortality found that studies with food industry ties more often have favourable results and conclusions compared with those with no industry ties, but the association was uncertain. ${ }^{16}$ One study has demonstrated an association of food industry sponsorship with the magnitude of effect estimates. ${ }^{17}$ In this examination, studies of soft drink consumption sponsored by the food industry reported significantly smaller harm effect estimates than those with no food industry sponsorship. A recent dairy industry-funded meta-analysis of observational studies found that studies without food industry sponsorship showed that dairy consumption was associated with a statistically significant decreased risk of developing CVD and type 2 diabetes, while studies with food industry sponsorship did not. ${ }^{18}$

The primary objective of this systematic review and meta-analysis is to determine whether:

- Studies of observational design examining the associations of dairy foods with CVD with food industry ties (industry sponsorship and/or authors with a COI) are more likely to have results and/or conclusions that are favourable to industry than those with no industry ties.

The secondary objectives of this review are to determine whether observational studies with food industry ties compared with no industry ties:

1. Differ in their risk of bias.

2. Have a higher level of discordance between study results and conclusions, with the conclusions more likely to be favourable compared with the results.

\section{METHODS}

We conducted a systematic review of observational studies examining the effect of dairy consumption on CVD. Our study is registered with PROSPERO (see online supplemental file 1). ${ }^{19}$

\section{Search strategy}

The search included terms to locate observational studies and randomised controlled trials, the latter of which are for a separate systematic review. The search used was based on the Process Manual used to develop the 2013 Australian Dietary Guidelines and the guidance of an information specialist. ${ }^{20}$ The search dates used were to ensure that we identified the studies used to inform the recommendations in these guidelines. We therefore searched the following databases from January 2000 to February 2019: MEDLINE; CINAHL; PubMed; PreMEDLINE; Cochrane Library; PsycINFO; Science Direct and ERIC. The search strategy used for Ovid MEDLINE on 1 February 2019 is shown in online supplemental file 2. We adapted this strategy for the other databases. We hand searched reference lists of the identified studies and reviews.

\section{Eligibility criteria}

We included studies of cohort or case-control designs that estimated the effects of dairy consumption on CVD outcomes in healthy adults. We focused on these study designs as they are often used to assess the association of diet with long-term health outcomes.

We included studies with no restriction on the authors' definition of dairy. For example, some authors' defined dairy as milk, yoghurt and cheese, while others defined dairy as 'whole fat' milk, yoghurt and cheese. We included studies that compared dairy foods with other foods or compared various levels of dairy consumption.

We included studies that measured any clinical outcome of CVD, defined as either mortality related to specific CVD events, and/or CVD events, (eg, first myocardial infarction, total stroke and so on) or incidence of elevated blood pressure/hypertension.

We excluded conference presentations, opinion pieces and letters to the editor. We had no language restrictions.

\section{Types of outcome measures}

\section{Primary outcomes}

We hypothesised that studies with food industry sponsorship and/or authors with a COI with the food industry would be more likely to have favourable findings than those with no industry ties. We assessed three primary outcomes:

\section{Statistical significance of results favourable to dairy}

Favourable results were defined as those that were in the direction of showing a health benefit of dairy product(s), and were statistically significant at the 0.05 level (two tailed), such as a statistically significant decreased risk of CVD compared with the comparator (ie, another food or lower dairy consumption). Otherwise, results were classified as unfavourable. In the circumstance where a study reported multiple results (eg, first myocardial infarction and total stroke), only one result needed to be 'favourable' for the study as a whole to be classified as 'favourable'. 
Effect size of results

Effect size was defined as the risk ratio (RR), hazard ratio (HR) or odds ratio (OR) between dairy foods tested versus comparator on the CVD outcome.

\section{Conclusions}

Conclusions that suggested that the dairy consumption was beneficial to health by decreasing CVD were considered favourable. Otherwise, the conclusions were considered unfavourable. In the circumstance where a study reported multiple results (eg, first myocardial infarction and total stroke), only one conclusion needed to be 'favourable' for the study as a whole to be classified as 'favourable'.

\section{Secondary outcomes}

We assessed two secondary outcomes:

\section{The risk of bias of the included studies}

To evaluate the risk of bias of included observational studies, we used an adapted version of the Cochrane Collaboration's 'Risk of Bias in Non-Randomised Studies-of Interventions' (ROBINS-I) tool, ${ }^{21}$ the ROBINS-of Exposure (ROBINS-E). ${ }^{22}$ Bias is assessed across seven domains ('bias due to confounding', 'bias in selection of participants', 'bias in classification of exposures', 'bias due to deviations from exposures', 'bias due to missing data', 'bias in measurement of outcomes', 'bias in selection of reported results'), with each domain classified low, moderate, serious, critical risk of bias or no information. The first step in using the ROBINS-E tool is to identify all possible confounders that a study should control. We developed this list of confounders by searching the literature for the most recent systematic reviews on possible confounders and having this list reviewed by expert professors in nutrition at The University of Sydney (see online supplemental file 3 for the list of confounders). An overall risk of bias rating for the study is given based on the domain with the highest risk of bias rating. For example, if a study is rated as being at a 'critical' risk of bias in one domain, the overall risk of bias rating is 'critical.' In the circumstance where a study reported multiple results (eg, stroke and myocardial infarction), the risk of bias was only assessed for one randomly selected outcome.

\section{Concordance between study results and conclusions}

Results unfavourable to the sponsor with conclusions favourable to the sponsor were considered discordant. Otherwise, the results and conclusions were considered concordant.

\section{Selection of studies}

Three investigators ( $\mathrm{NC}, \mathrm{SM}$ and $\mathrm{AF}$ ), working independently in pairs, screened the titles and abstracts of all records for obvious exclusions. If both investigators agreed on excluding the study, the full text was not retrieved. Three investigators (NC, SM and AF) working independently in pairs, assessed the full text of potentially eligible studies against the inclusion criteria. If agreement could not be reached, a fourth investigator (LB) resolved the conflict.

\section{Selection of results for meta-analysis}

If total dairy consumption had been assessed in the study, we included this as our only exposure. If total dairy consumption had not been assessed, we included any type of dairy consumption (eg, milk, yoghurt and cheese; or low fat, high fat) other than fermented milk as our exposure. We included the results comparing the highest level of dairy consumption to the lowest level of dairy consumption (eg, 'yes' to dairy consumption vs 'no' to dairy consumption, tertile 3 vs tertile 1 , quartile 4 vs quartile 1 , quintile 5 vs quintile 1 ). For the meta-analyses if our prespecified rules for selecting results did not allow us to uniquely identify one exposure for inclusion, we randomly selected one result.

If 'CVD mortality/death/s' (verbatim) had been assessed, we included this as our only outcome. If not, we included any type of CVD mortality (eg, coronary heart disease mortality, stroke mortality and so on) as our outcome. If there were no mortality outcomes assessed in the study, we included any CVD event or incidence of elevated blood pressure/hypertension as our outcome. If a study used a composite outcome, which was a combination of multiple outcomes, the result pertaining to the composite outcome was selected. For the meta-analyses if our prespecified rules for selecting results did not allow us to uniquely identify one outcome for inclusion, we randomly selected one result.

\section{Data collection}

From each study we extracted:

- Year of publication.

- Study design (cohort or case-control).

- Sample size of study.

- Age of participants (combined or if reported, separately).

- Exposure duration or observation period.

- How the study defined dairy (verbatim).

- Disclosure of funding source (no disclosure, yes and there is a sponsor, the authors state they received no funding for their work).

- Name of the funders of the study (verbatim).

- Role of the funders (role of the sponsor not mentioned, sponsor not involved in study design and analyses, sponsor involved, not applicable).

- Disclosure of author COI (no disclosure, yes (if at least one author had a COI), the authors state they had no conflicts of interest to declare).

- Authors' COI statement (verbatim).

- Outcomes assessed in the study (any CVD death and/ or event or blood pressure/hypertension).

- The numerical results of the study (eg, OR, HR, RR). All extracted data from the included studies were stored in REDcap, a secure web-based application for the collection and management of data. ${ }^{23}$ Five investigators (NC, SM, AF, AL and JD) working independently in pairs 
extracted data from the included studies. Discrepancies in data extraction were resolved by consensus. If agreement could not be reached, a sixth investigator (LB) resolved the discrepancy.

\section{Classification of industry sponsorship and author COI}

Sponsorship was categorised as (1) industry or (2) nonindustry. Industry-sponsored studies were defined as those that declared any sponsorship from the food industry, including 'Big Food' (ie, Danone, Kraft, Unilever and so on), trade associations (ie, dairy associations and organisations) and dairy industry (ie, primary producers). Studies with food industry sponsorship plus any other sponsorship were classified as industry. Any study that did not contain a funding disclosure statement was classified as 'non-industry'.

Studies with at least one author with any disclosed financial tie with the food industry were classified as having a COI. Author COI were categorised as (1) COI or (2) no COI. Studies with no authors with disclosed financial ties with the food industry were classified as 'no COI'.

Since the number of studies with industry sponsorship or author COI was small, we also categorised studies as having 'industry ties' for analysis. Studies classified as having an industry tie were industry sponsored and/or had an author COI. Otherwise, they were classified as having no industry ties.

\section{Analysis}

We report the frequencies and percentages of the study characteristics across all studies, and separately, by sponsorship, COI and industry ties. We visually present the risk of bias rating for each domain and overall across each study.

To quantify the association between industry ties, food industry sponsorship, or authors with a COI with the food industry and (1) favourable results, (2) favourable conclusions, (3) overall risk of bias across each study and (4) level of concordance, we calculated RR (and 95\% CIs). To analyse the risk of bias rating for each study, we dichotomised the overall risk of bias ratings as low (low or moderate) or high (serious or critical).

We conducted meta-analysis to examine whether studies with food industry ties, food industry sponsorship or authors with a COI with the food industry modified the magnitude of effect of dairy on CVD outcomes. For each outcome, we combined effect estimates using a randomeffects meta-analysis model using the inverse variance method. DerSimonian and Laird's method of moments estimator was used to estimate between study heterogeneity. We fitted separate meta-analyses for studies that had measured the association using HRs and those that had used either RRs or ORs. It is not recommended to combine HRs with RRs and ORs in a meta-analysis, as HRs represent instantaneous risk over the study time period, whereas RRs and ORs estimate risk/odds at a fixed time point. ${ }^{24}$ We considered that the ORs approximated RRs given CVD events were rare.

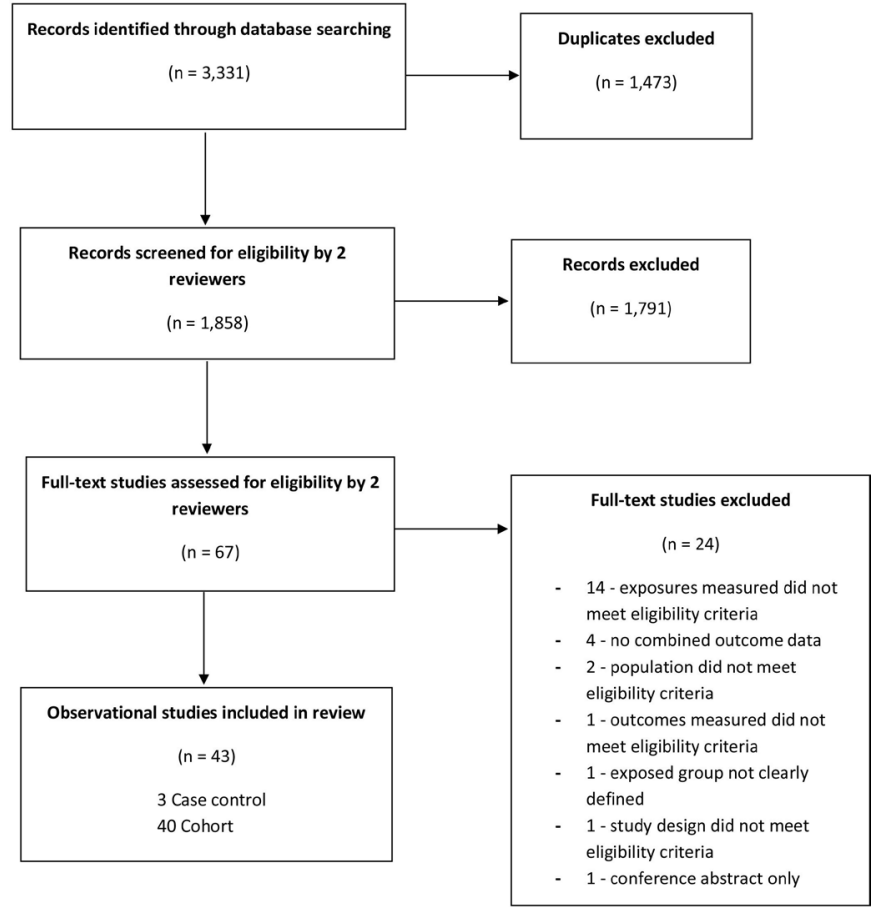

Figure 1 Study flow diagram.

We undertook a fixed-effects test for subgroup differences (defined by industry sponsorship/authors COI) using the $X^{2}$ test and calculated the ratio of RRs (ORs) or HRs along with 95\% CIs. Analyses were undertaken in Review Manager V.5.3. ${ }^{25}$

We planned to use sensitivity analysis to assess the influence of risk of bias by restricting the analysis to studies at 'low risk of bias' overall (ie, an overall risk of bias rating of low or moderate). However, as the overall risk of bias was high across all studies, this was not undertaken.

\section{Patient and public involvement}

No patient involved.

\section{RESULTS}

As shown in figure 1 , there were 1858 studies screened for inclusion and 43 studies were included (3 case-controls, 40 cohorts). See online supplemental file 4 for 'list of excluded studies and reasons for exclusion'.

\section{Characteristics of included studies}

All studies were published between 2001 and 2019. All but one contained a funding disclosure. Eight studies disclosed food industry sponsorship, but only two of these studies described the role of the sponsor. Six studies did not contain an author COI disclosure statement. Ten studies contained an author with a COI with the food industry. Fourteen studies were classified as having industry ties, disclosing food industry sponsorship and/ or an author with a COI.

As shown in table 1, most characteristics were similarly distributed across studies with industry ties or no industry ties. Studies with industry ties (64\%) were more likely to 
Table 1 Characteristics of the included studies by sponsorship, author conflict of interest (COI) and industry ties.

Funding source, $\mathbf{n}(\%)^{*}$

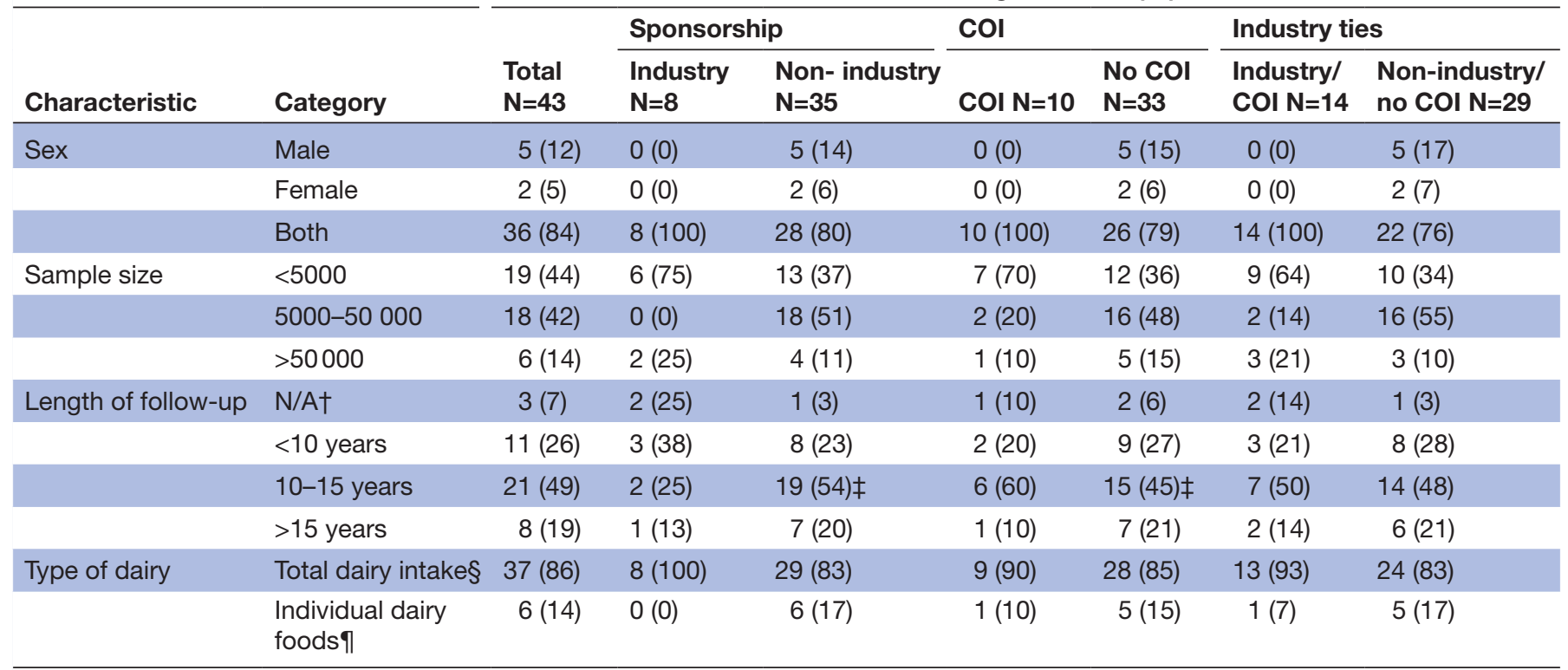

${ }^{*}$ Percentages may not add to 100 due to rounding.

†Follow-up is not applicable for case-control studies.

fFollow-up for Johansson described the follow-up as '8-12 years', we took the median of 10 years.

$\S$ This includes studies that looked at nutrients for example, calcium, fat and protein by measuring total dairy intake.

IIndividual foods included milk, cheese and yoghurt.

$\mathrm{N} / \mathrm{A}$, not available.

have sample sizes $<5000$ than non-industry-sponsored studies $(34 \%)$. A greater proportion of industrysponsored studies $(100 \%)$ than non-industry-sponsored studies $(83 \%)$ focused on total dairy intake rather than a specific food. Details of the individual studies are in online supplemental file 5 .

\section{Risk of bias in included studies}

Every study was classified as having an overall high risk of bias, with 10 assessed as having a serious risk of bias and 33 as having a critical risk of bias (figure 2). Most studies were assessed as having a critical risk of bias rating for the domain 'bias due to confounding'. An example of one of the several confounders we identified that studies needed to control for was fruit and vegetable intake. If these confounders were not controlled for appropriately when measuring the effect of dairy intake on a CVD outcome, the study was classified as having a risk of bias for the confounding domain.

Studies without industry ties or without an author with a COI were more likely to have a serious or critical risk of bias rating for 'bias in classification of exposures'. For example, if a study did not use a validated food frequency questionnaire to measure the dietary intake of dairy, the study was classified as having a risk of bias for the domain of classification of exposures. For all other domains, the risk of bias classifications were similarly distributed across studies with industry ties, industry sponsorship or COI versus no industry ties, industry sponsorship or COI, respectively (see online supplemental file 6).
Favourable results—statistical significance: industry ties versus no industry ties; industry sponsorship versus no industry sponsorship; COI versus no COI

There was no clear evidence of an association between the reporting of favourable results and studies with industry ties $(1 / 14)$ compared with those with no industry ties $(8 / 29), \mathrm{RR}=0.26(95 \%$ CI 0.04 to $1.87 ; \mathrm{n}=43$ studies) (online supplemental file 7 ). When comparing studies with industry sponsorship $(1 / 8)$ with those with no industry sponsorship $(8 / 35)$, there was no clear evidence of an association, $\mathrm{RR}=0.55$ (95\% CI 0.08 to 3.77 ; $\mathrm{n}=43$ studies). There was again no clear evidence of an association between the reporting of favourable results and studies with an author with a COI $(0 / 10)$ than those with no $\operatorname{COI}(9 / 33), \mathrm{RR}=0.16(95 \%$ CI 0.01 to $2.57 ; \mathrm{n}=43$ studies).

Effect size, CVD: industry ties versus no industry ties; industry sponsorship versus no industry sponsorship; COI versus no COI

For studies that quantified the association between dairy consumption and CVD outcomes using an RR, we found no important difference in the magnitude of the effect in studies with industry ties ( $R R=0.89 ; n=3$ studies) compared with those studies with no industry ties, $(\mathrm{RR}=0.99 ; \mathrm{n}=7$ studies) (ratio of RRs 0.90 (95\% CI 0.74 to 1.09$) ; \mathrm{p}=0.27$ ) (online supplemental file 8 ). For studies that had quantified the association using HRs, we similarly did not find an important difference in the magnitude of HRs between studies with industry ties, $(\mathrm{HR}=0.96 ; \mathrm{n}=7$ studies $)$ 


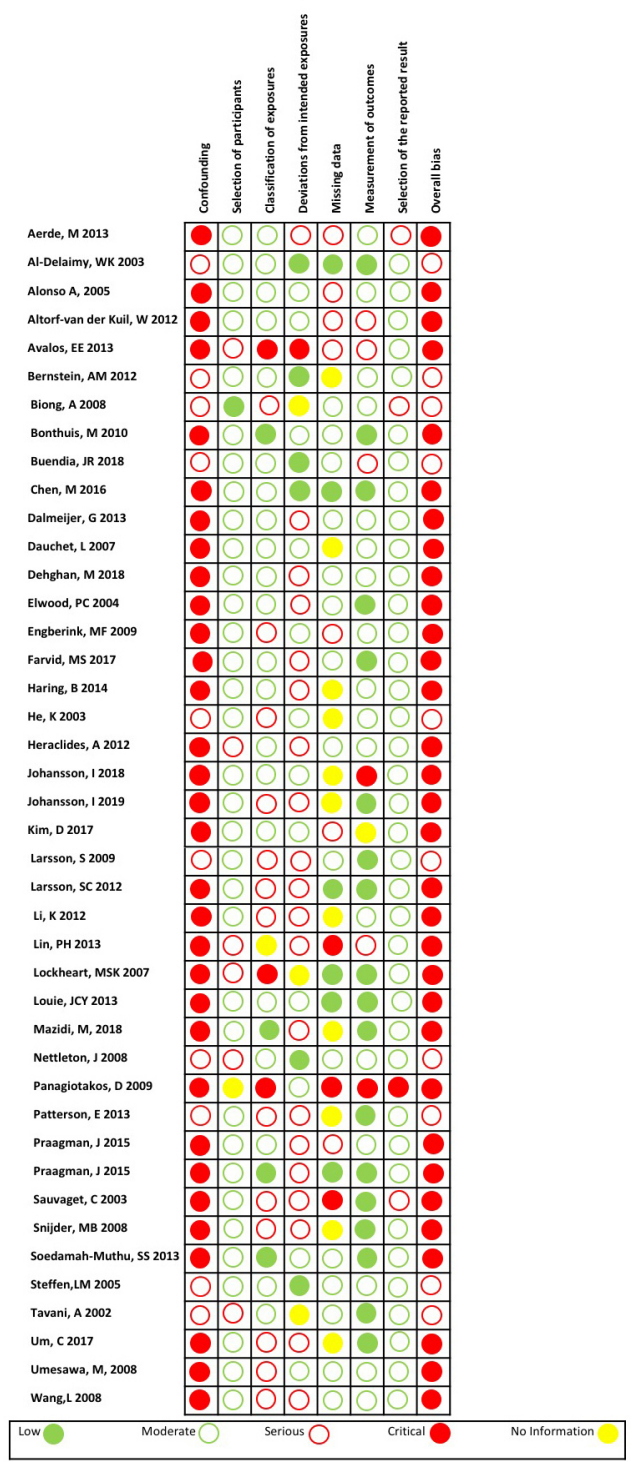

Figure 2 Risk of bias in included studies.

and those studies with no industry ties, $(\mathrm{HR}=0.95 ; \mathrm{n}=14$ studies) (ratio of HRs 1.01 (95\% CI 0.90 to 1.13 ); $\mathrm{p}=0.86$ ).

In our analysis comparing studies with industry sponsorship, (RR 0.83; $\mathrm{n}=2$ studies) and those with no industry sponsorship (RR $0.97 ; n=8$ studies), we again did not find an important difference in the magnitude of RRs (ratio of RRs 0.86 (95\% CI 0.44 to 1.66); $\mathrm{p}=0.65$ ) (online supplemental file 8). However, when we compared industry-sponsored studies, ( $\mathrm{HR}=0.78 ; \mathrm{n}=3$ studies $)$ and non-industry-sponsored studies, (HR=0.97; $\mathrm{n}=18$ studies) that measured the association using HRs, we found a statistically significant difference in the magnitude of the HRs (ratio of HRs 0.80 (95\% CI 0.66 to 0.97 ); $\mathrm{p}=0.03$ ) (figure 3).

In our analysis comparing studies with an author with a COI (RR 0.89; $\mathrm{n}=2$ studies) and those with no COI, (RR $0.99 ; \mathrm{n}=8$ studies), we found no important difference in the magnitude of RRs (ratio of RRs 0.90 (95\% CI 0.76 to 1.07 ); $\mathrm{p}=0.22$ ) (online supplemental file 8 ). When we compared studies with a COI, $(\mathrm{HR}=1.00 ; \mathrm{n}=5$ studies $)$ and studies with no COI, (HR=0.93; $\mathrm{n}=16$ studies $)$ that measured the association using HRs, we again found no difference in the magnitude of the HRs (ratio of HRs 1.08 (95\% CI 0.99 to 1.17$) ; \mathrm{p}=0.12$ ).

Effect size, elevated blood pressure/hypertension: industry ties versus no industry ties, and industry sponsorship versus no industry sponsorship

We found no important difference in the magnitude of the HRs for elevated blood pressure/hypertension in studies with industry ties, $(\mathrm{HR}=0.89 ; \mathrm{n}=2)$ and those studies with no industry ties, $(\mathrm{HR}=0.78 ; \mathrm{n}=5)$ (ratio of HRs 1.14 (95\% CI 0.88 to 1.49 ); $\mathrm{p}=0.32$ ) (online supplemental file 8 ).

All of these studies with industry ties also had industry sponsorship, so the ratio of HRs was the same.

Favourable conclusions: industry ties versus no industry ties; industry sponsorship versus no industry sponsorship; COI versus no $\mathrm{COI}$

There was no clear evidence of an association between the reporting of favourable conclusions and studies with industry ties (4/14) compared with those with no industry ties $(11 / 29), \mathrm{RR}=0.75$ (95\% CI 0.29 to $1.95 ; \mathrm{n}=43$ ) (online supplemental file 7). When we compared studies only by industry sponsorship, there was no clear evidence of an association between industry-sponsored studies $(3 / 8)$, compared with studies with no sponsorship (12/35), $\mathrm{RR}=1.09$ ( $95 \%$ CI 0.40 to $2.99 ; \mathrm{n}=43$ ). There was again no clear evidence of an association between the reporting of favourable conclusions and studies with an author with a COI $(2 / 10)$ than those without a COI $(13 / 33), R R=0.51$ (95\% CI 0.14 to $1.88 ; \mathrm{n}=43$ studies).

\section{Risk of bias assessment by industry ties}

As every study had an overall high (serious or critical) risk of bias rating, there was no difference in the proportion of studies at a high risk of bias between those with industry ties, industry sponsorship or COI and those without industry ties, sponsorship or COI.

\section{Concordance between study results and conclusions}

Six (of 43) studies, all with unfavourable results, overemphasised the benefits of the dairy exposure in their conclusions and thus were coded as 'favourable' conclusions.

There was no clear evidence of an association between discordant results and conclusions and studies with industry ties $(3 / 14)$ than those with no industry ties $(3 / 29), \mathrm{RR}=2.07$ (95\% CI 0.48 to $8.99 ; \mathrm{n}=43$ ) (online supplemental file 7 ). There was no clear evidence of an association when comparing studies with industry sponsorship $(2 / 8)$ with those with no industry sponsorship (4/35), $R R=2.19$ (95\% CI 0.48 to 9.94). There was again no clear evidence of an association between studies with an author with a COI $(2 / 10)$ than those with no COI (4/33), RR=1.65 (95\% CI 0.35 to 7.72; $\mathrm{n}=43$ ). 


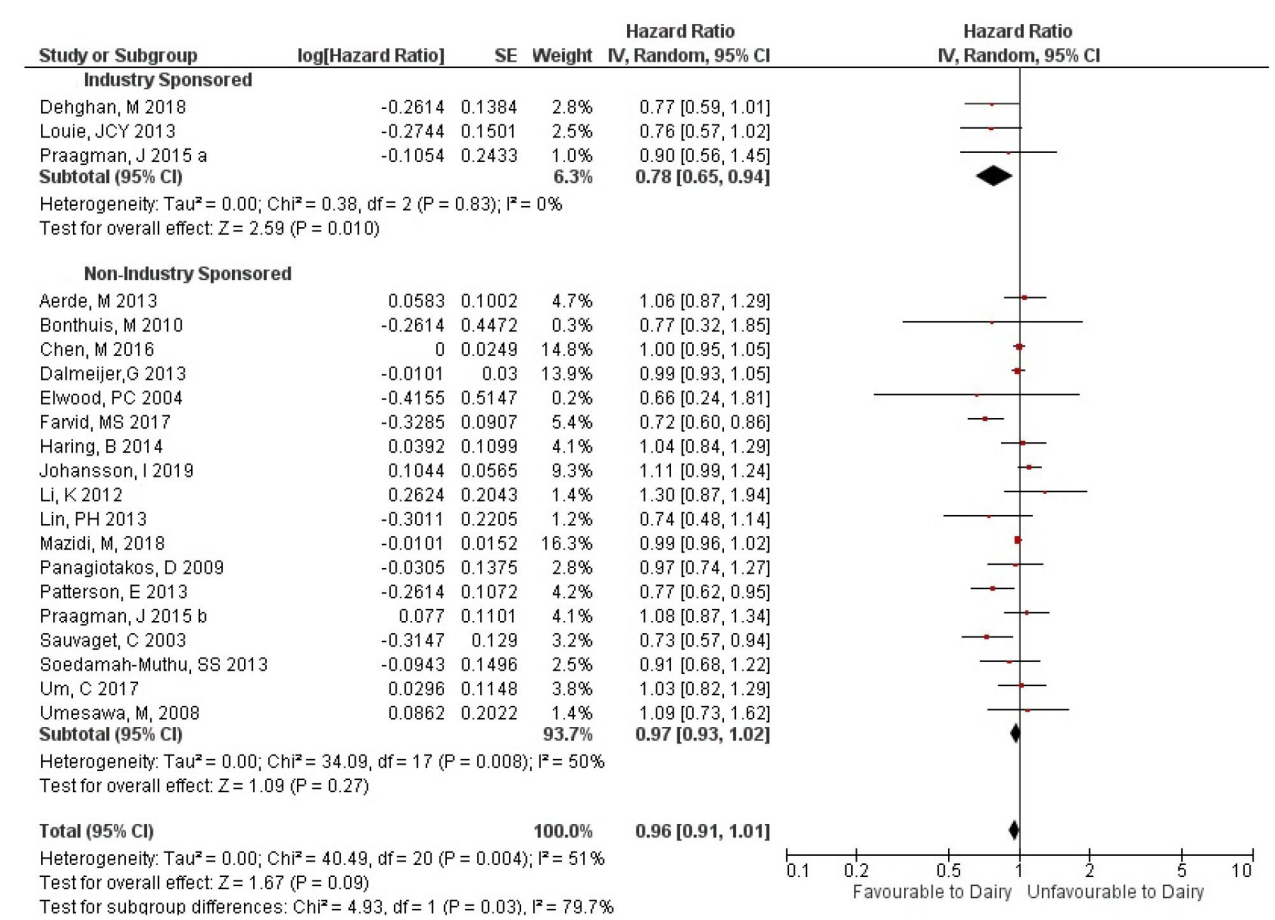

Figure 3 Effect size, cardiovascular disease: industry sponsorship versus no industry sponsorship, HR.

\section{DISCUSSION}

There was no clear evidence of an association between studies with food industry ties and the reporting of favourable results and conclusions of observational studies measuring the associations of dairy foods with CVD outcomes. The 'mixed' group of funders we identified in the industry-sponsored studies may influence these results, as the funding effect may be diluted by this heterogeneous group of sponsors. Unlike in drug studies, ${ }^{12}$ the funders in the studies included in this review were extremely diverse, with Big Food and trade association jointly sponsoring several studies. Thus, dairy foods are not their sole interest.

The meta-analysis of HRs of CVD outcomes found that studies with industry sponsorship showed a greater benefit from dairy than studies without industry sponsorship, and this difference was statistically significant. The meta-analysis of RRs of CVD outcomes found a similar estimate; however, this was not statistically significant. The likely reason for this was that the meta-analysis of RRs had fewer studies, and so the ratio of RRs could not be as precisely estimated. We found no evidence of a clinically important difference in the magnitude of effect between studies with industry ties or authors with a COI compared with those with no industry ties or no COI for other outcomes.

For every study, the overall risk of bias was classified as high (meaning either serious or critical). Therefore, differences in the risk of bias across studies with and without industry ties would not seem to provide an explanation for our findings. However, the version of the ROBINS-E tool that we used may not have been able to adequately discriminate across the studies, as perhaps is indicated by the uniformity in risk of bias classification. ${ }^{26}$ Therefore, we cannot rule out the possibility that differences in bias across studies with and without industry ties may partly explain our findings.

\section{Strengths and limitations of this review}

Our review was prospectively registered in PROSPERO. ${ }^{19}$ We followed explicit inclusion and exclusion criteria, conducted a comprehensive search across multiple databases and hand searched reference lists for the included studies.

For those studies missing a funding or author COI disclosure, we did not contact the authors and we therefore may be underestimating the number of studies with industry ties. The tool that we used to assess the risk of bias is still under development, however it is unlikely any future changes to the tool will affect the risk of bias ratings. ${ }^{22}$ We did not analyse studies of low-fat and full-fat dairy or other types of dairy products separately. Industry ties may have different effects on studies of low-fat or full-fat dairy foods or other foods and drinks. A final limitation of our study is that we relied on definitions of exposures and outcomes that were used in the original studies included in our analyses. Using finer categorisations of exposures and outcomes would not provide a sufficient sample size to do our analyses. However, future studies, using additional data and finer categorisations, may have different results.

Agreements and disagreements with other studies or reviews The observed greater benefit of dairy on CVD outcomes in industry-sponsored studies compared with non-industrysponsored studies corroborates previous research that 
has demonstrated studies sponsored by the food industry reported smaller harmful effect sizes for soft drink consumption, compared with non-industry-sponsored studies. ${ }^{17}$ It is not consistent, however, with a recent metaanalysis funded by the Israel Dairy Board that found non-statistically significant differences in the estimated associations between industry-funded and non-industryfunded studies. ${ }^{18}$ The differences in the results of our current review and this previous study can be attributed to a number of important factors in how the studies were conducted, including how the exposures were classified, the outcomes selected for the meta-analyses and the analysis method used. For the exposures, our review included yoghurt and cheese, as well as 'total dairy' and milk, whereas the Dairy Board study included only 'total dairy' and milk as exposures. We included all outcomes related to CVD, and the Dairy Board study included only CVD and stroke, as well as type 2 diabetes. For the analysis method, we fitted separate meta-analyses for studies that had measured the association using HRs and those that had used either RRs or ORs, while the Dairy Board study only measured the associations using RRs.

The lack of difference in the risks of bias between studies with industry ties and those with no industry ties, is consistent with a previous review that examined the association of industry ties with outcomes of studies examining the effect of wholegrain foods on CVD and mortality that used the same tool to assess risk of bias. ${ }^{16}$ These findings have also been shown in pharmaceutical, tobacco and nutrition research that have demonstrated industry-sponsored studies are of equal or better internal validity than studies with no sponsorship. ${ }^{12} 13152728$

\section{Implications for clinicians, policymakers and future research}

As dietary guidelines depend on an evidence base that should be as free as possible of bias, the difference in the magnitude of effects between industry-sponsored studies compared with non-industry-sponsored studies is concerning. Therefore, the dairy intake recommendations made in dietary guidelines should account for the potential influence of industry sponsorship on evidence of health effects. Nutrition studies included in systematic reviews used in the development of dietary guidelines should be assessed using empirical methods to identify factors associated with study results. Current risk of bias tools should therefore be amended or supplemented to include industry sponsorship and author COI as a separate risk of bias domain. The University of California, San Francisco's Navigation Guide assesses both author COI and funding sources as a risk of bias in human and animal studies. ${ }^{29}$ As the study designs used in nutrition are the same as those used to evaluate the harms of an exposure in environmental health, dietary guideline committees could consider adopting this tool to evaluate the risk of bias of the studies included in the systematic reviews used to develop dietary guidelines.

Industry sponsors may bias research via different mechanisms, including the design and conduct of a study, the selective reporting of results, how they code events, analyse data, by spinning conclusions, ${ }^{11}$ as well as framing how the questions are asked. ${ }^{30-32}$ It has been suggested that the dairy industry may preferentially fund research on topics which will provide them with more favourable outcomes. ${ }^{33}$ The influence of the food industry on the research agenda has been demonstrated in an examination of research topics covered by samples of randomised controlled trials included in systematic reviews of nutrition studies and obesity. ${ }^{34}$ It was shown that most food industry studies focused on the manipulations of specific nutrients, and not on dietary behaviours, therefore limiting the public health relevance of rigorous evidence available for use in both systematic reviews and dietary guidelines. ${ }^{34}$ The topics examined in cohort studies on the relationship of nutrition and obesity, which tend to focus on more complex exposures than trials, did not demonstrate a similar influence of funding source. However, the disclosure of food industry sponsorship was low, making a comparison difficult. ${ }^{35}$

This present study has also demonstrated that there is significant funding for nutrition research that comes from non-industry sources, including academia and government. In this study, only 8 studies had food industry sponsorship, while 34 had a non-food industry sponsorship. A similar rate was seen in a study that assessed the association of industry ties with outcomes of studies examining the effect of wholegrain foods on CVD and mortality, with only 5 industry-sponsored studies and 17 non-industrysponsored studies. ${ }^{16}$ To eliminate this risk of bias from nutrition research, investigators should use only nonindustry sources to fund their research.

\section{CONCLUSION}

There was no clear evidence of an association between studies with food industry ties and the reporting of favourable results and conclusions compared with studies without industry ties. However, the statistically significant difference in the magnitude of effects identified in industry-sponsored studies compared with non-industrysponsored studies is important in quantifying industry influence on studies included in dietary guidelines.

\section{Author affiliations}

${ }^{1}$ Charles Perkins Centre, The University of Sydney, Sydney, New South Wales, Australia

${ }^{2}$ Centre for Evidence-Based Medicine Odense (CEBM0), Odense University Hospital, University of Southern Denmark, Odense, Denmark

${ }^{3}$ Faculty of Medicine and Health, The University of Sydney, Sydney, New South Wales, Australia

${ }^{4}$ School of Public Health and Preventive Medicine, Monash University, Melbourne, Victoria, Australia

Acknowledgements We thank Agnes Lau, University of California, San Francisco, for her assistance with data collection.

Contributors NC, AF and LB designed and wrote the review protocol. NC wrote the search strategy and undertook the literature search. NC, AF and SM conducted the title and abstract screening and full-article screening for final study inclusion. NC, AF, JD, AL and SM conducted data collection and cleaning, LB supervised. NC and 
JM undertook all data analysis. LB advised on methods, statistical analyses and interpretation of findings. All authors contributed to the final manuscript. NC and LB are guarantors.

Funding This work was supported by Australian Health and Medical Research Council Project Grant APP 1139997. NC is a recipient of the James Millner PhD Scholarship in Pharmacy from the University of Sydney.

Competing interests None declared.

Patient consent for publication Not required.

Provenance and peer review Not commissioned; externally peer reviewed.

Data availability statement Data are available upon reasonable request. Available from The University of Sydney data repository. DOI to be determined.

Supplemental material This content has been supplied by the author(s). It has not been vetted by BMJ Publishing Group Limited (BMJ) and may not have been peer-reviewed. Any opinions or recommendations discussed are solely those of the author(s) and are not endorsed by BMJ. BMJ disclaims all liability and responsibility arising from any reliance placed on the content. Where the content includes any translated material, BMJ does not warrant the accuracy and reliability of the translations (including but not limited to local regulations, clinical guidelines, terminology, drug names and drug dosages), and is not responsible for any error and/or omissions arising from translation and adaptation or otherwise.

Open access This is an open access article distributed in accordance with the Creative Commons Attribution Non Commercial (CC BY-NC 4.0) license, which permits others to distribute, remix, adapt, build upon this work non-commercially, and license their derivative works on different terms, provided the original work is properly cited, appropriate credit is given, any changes made indicated, and the use is non-commercial. See: http://creativecommons.org/licenses/by-nc/4.0/.

\section{ORCID iDs}

Nicholas Chartres http://orcid.org/0000-0002-9659-6739

Lisa Bero http://orcid.org/0000-0003-1893-6651

\section{REFERENCES}

1 Qin L-Q, Xu J-Y, Han S-F, et al. Dairy consumption and risk of cardiovascular disease: an updated meta-analysis of prospective cohort studies. Asia Pac J Clin Nutr 2015;24:90-100.

2 Alexander DD, Bylsma LC, Vargas AJ, et al. Dairy consumption and CVD: a systematic review and meta-analysis. Br J Nutr 2016;115:737-50.

3 Gholami F, Khoramdad M, Esmailnasab N, et al. The effect of dairy consumption on the prevention of cardiovascular diseases: a meta-analysis of prospective studies. J Cardiovasc Thorac Res 2017:9:1-11.

4 Drouin-Chartier J-P, Brassard D, Tessier-Grenier M, et al. Systematic review of the association between dairy product consumption and risk of Cardiovascular-Related clinical outcomes. Adv Nutr 2016;7:1026-40.

5 Lee M, Lee H, Kim J. Dairy food consumption is associated with a lower risk of the metabolic syndrome and its components: a systematic review and meta-analysis. Br J Nutr 2018;120:373-84.

6 National Health and Medical Research Council. Australian Dietary Guidelines. Canberra: National Health and Medical Research Council, 2013.

7 Public Health England. The Eatwell Guide [online], 2016. Available: https://www.gov.uk/government/publications/the-eatwell-guide [Accessed 18 Mar 2016].

8 Lexchin J. Those who have the gold make the evidence: how the pharmaceutical industry biases the outcomes of clinical trials of medications. Sci Eng Ethics 2012;18:247-61.

9 Sismondo S. How pharmaceutical industry funding affects trial outcomes: causal structures and responses. Soc Sci Med 2008;66:1909-14.

10 Boutron I, Dutton S, Ravaud P, et al. Reporting and interpretation of randomized controlled trials with statistically nonsignificant results for primary outcomes. JAMA 2010;303:2058-64.

11 Odierna DH, Forsyth SR, White J, et al. The cycle of bias in health research: a framework and toolbox for critical appraisal training. Account Res 2013:20:127-41.

12 Lundh A, Lexchin J, Mintzes B, et al. Industry sponsorship and research outcome. Cochrane Database Syst Rev 2017;2:Mr000033.
13 Barnes DE, Bero LA. Industry-funded research and conflict of interest: an analysis of research sponsored by the tobacco industry through the center for indoor air research. J Health Polit Policy Law 1996;21:515-42.

14 Yank V, Rennie D, Bero LA. Financial ties and concordance between results and conclusions in meta-analyses: retrospective cohort study. BMJ 2007;335:1202-5.

15 Chartres N, Fabbri A, Bero LA. Association of industry sponsorship with outcomes of nutrition studies: a systematic review and metaanalysis. JAMA Intern Med 2016;176:1769-77.

16 Chartres N, Fabbri A, McDonald S, et al. Association of industry ties with outcomes of studies examining the effect of wholegrain foods on cardiovascular disease and mortality: systematic review and meta-analysis. BMJ Open 2019;9:e022912.

17 Vartanian LR, Schwartz MB, Brownell KD. Effects of soft drink consumption on nutrition and health: a systematic review and metaanalysis. Am J Public Health 2007;97:667-75.

18 Mishali M, Kisner M, Avrech T. Funding sources and outcomes of dairy consumption research - a meta-analysis of cohort studies: the case of type-2 diabetes and cardiovascular diseases. Int Dairy $J$ 2019.

19 National Institute for Health Research. International prospective register for sytematic reviews [online], 2015. Available: http://www. crd.york.ac.uk/PROSPERO/ [Accessed 11 Mar 2016].

20 Dietitians Association of Australia. A review of the evidence to address targeted questions to inform the revision of the Australian dietary guidelines 2009: process manual, 2011.

21 Sterne JA, Hernán MA, Reeves BC, et al. ROBINS-I: a tool for assessing risk of bias in non-randomised studies of interventions. BMJ 2016;355:i4919.

22 University of Bristol. The ROBINS-E tool (Risk Of bias in nonrandomized studies - of exposures), 2019. Available: https://www. bristol.ac.uk/population-health-sciences/centres/cresyda/barr/ riskofbias/robins-e/

23 Harris PA, Taylor R, Thielke R, et al. Research electronic data capture (REDCap)--a metadata-driven methodology and workflow process for providing translational research informatics support. J Biomed Inform 2009;42:377-81.

24 Tierney JF, Stewart LA, Ghersi D, et al. Practical methods for incorporating summary time-to-event data into meta-analysis. Trials 2007;8:16.

25 The Cochrane Collaboration. Review Manager (RevMan) [Computer program]. Version 5.3. Copenhagen: The Nordic Cochrane Centre, 2014.

26 Bero L, Chartres N, Diong J, et al. The risk of bias in observational studies of exposures (ROBINS-E) tool: concerns arising from application to observational studies of exposures. Syst Rev 2018;7:242

27 Mandrioli D, Kearns CE, Bero LA. Relationship between research outcomes and risk of bias, study sponsorship, and author financial conflicts of interest in reviews of the effects of artificially sweetened beverages on weight outcomes: a systematic review of reviews. PLoS One 2016;11:e0162198.

28 Cho MK, Bero LA. The quality of drug studies published in symposium proceedings. Ann Intern Med 1996;124:485-9.

29 Woodruff TJ, Sutton P. The navigation guide systematic review methodology: a rigorous and transparent method for translating environmental health science into better health outcomes. Environ Health Perspect 2014;122:1007-14.

30 Fabbri A, Lai A, Grundy Q, et al. The influence of industry sponsorship on the research agenda: a scoping review. Am J Public Health 2018;108:e9-16.

31 Psaty BM, Prentice RL. Minimizing bias in randomized trials: the importance of blinding. JAMA 2010;304:793-4.

32 Psaty BM, Kronmal RA. Reporting mortality findings in trials of rofecoxib for Alzheimer disease or cognitive impairment: a case study based on documents from rofecoxib litigation. JAMA 2008;299:1813-7.

33 Wilde P, Morgan E, Roberts J, et al. Relationship between funding sources and outcomes of obesity-related research. Physiol Behav 2012;107:172-5.

34 Fabbri A, Chartres N, Scrinis G, et al. Study sponsorship and the nutrition research agenda: analysis of randomized controlled trials included in systematic reviews of nutrition interventions to address obesity. Public Health Nutr 2017;20:1306-13.

35 Fabbri A, Chartres N, Bero LA. Study sponsorship and the nutrition research agenda: analysis of cohort studies examining the association between nutrition and obesity. Public Health Nutr 2017;20:3193-9. 